Meta

Journal des traducteurs

Translators' Journal

\title{
Réflexions sur la terminologie
}

\section{Dorothy Nakos-Aupetit}

Volume 25, numéro 2, juin 1980

URI : https://id.erudit.org/iderudit/001925ar

DOI : https://doi.org/10.7202/001925ar

Aller au sommaire du numéro

Éditeur(s)

Les Presses de l'Université de Montréal

ISSN

0026-0452 (imprimé)

1492-1421 (numérique)

Découvrir la revue

Citer cet article

Nakos-Aupetit, D. (1980). Réflexions sur la terminologie. Meta, 25(2), 254-256.

https://doi.org/10.7202/001925ar

Ce document est protégé par la loi sur le droit d'auteur. L’utilisation des services d'Érudit (y compris la reproduction) est assujettie à sa politique d'utilisation que vous pouvez consulter en ligne.

https://apropos.erudit.org/fr/usagers/politique-dutilisation/
Cet article est diffusé et préservé par Érudit.

Érudit est un consortium interuniversitaire sans but lucratif composé de l’Université de Montréal, l’Université Laval et l’Université du Québec à Montréal. Il a pour mission la promotion et la valorisation de la recherche. https://www.erudit.org/fr/ 


\title{
RÉFLEXIONS SUR LA TERMINOLOGIE
}

\author{
Discipline et recherches \\ Nomenclature, lexique, vocabulaire et terminologie \\ Lacunes des dictionnaires \\ Terminologie $=$ étude systématique ? \\ La «notion» en terminologie unilingue et comparée
}

Avant que M. Robert Dubuc ne donne sa définition de la terminologie dans la Banque des mots de $1977, \mathrm{n}^{\circ} 3$, aucun ouvrage français consulté ne définissait clairement la terminologie comme une discipline ${ }^{1}$.

En effet, d'après les sources traditionnelles, la terminologie était toujours associée à un ensemble de mots spécialisés comprenant parfois leur signification et était souvent confondue avec la nomenclature, le lexique, le vocabulaire.

Or, rappelons que la nomenclature est une simple liste d'appellations. Par exemple, la nomenclature des rues de Paris serait l'étude de termes comme avenue, boulevard, villa, ruelle, etc. (exemple fourni par M. Jean Darbelnet, professeur émérite de l'Université Laval). Par ailleurs, le lexique et le vocabulaire sont en quelque sorte de petits dictionnaires pratiques, souvent abrégés, recueillant des mots classés dans un certain ordre ${ }^{2}$. Le vocabulaire donne des définitions alors que le lexique n'en donne pas ${ }^{3}$. La discipline qui les étudie est la lexicologie. Comme un des aspects importants de la terminologie (discipline) est d'étudier et d'établir des terminologies (ensembles de mots spécialisés), il peut exister une confusion entre les deux. Bien qu'il soit possible de considérer la terminologie comme une branche de la lexicologie, il ne faut pas oublier que la terminologie est davantage axée sur les besoins techniques et scientifiques des clients. Les procédés et la présentation ne sont pas toujours identiques. Si le lexique et le vocabulaire demeurent fixes, du moins jusqu'à une réédition de l'ouvrage, les fiches des terminologues permettent une mise à jour constante des renseignements d'ordre technique. Les données entrées dans une banque de mots peuvent être modifiées, améliorées. La présentation de données terminologiques peut aussi prendre la forme de lexiques ou de vocabulaires, mais uniquement à la demande des intéressés.

1. Voici cette définition reprise dans le Manuel pratique de terminologie, Linguatech, 1978, du même auteur: "l'art de repérer, d'analyser et, au besoin, de créer le vocabulaire pour une technique donnée, dans une situation concrète de fonctionnement de façon à répondre aux besoins d'expression de l'usager. »

2. Explication reconstituée à partir de renseignements provenant du Dictionnaire des synonymes de Bénac, Hachette, 1956.

3. Le Vocabulaire systématique de la terminologie. Proposition relative à la révision de la recommandation ISO R 1087, Montréal, mai 1977 , cite les définitions suivantes:

«Vocabulaire: Répertoire, unilingue ou multilingue, qui inventorie les termes relatifs à un domaine, lesquels sont généralement présentés dans un ordre systématique et toujours suivi de leur définition.

Note: Des illustrations accompagnent ou remplacent parfois les définitions.»

«Lexique : Répertoire qui présente une liste limitée de termes appartenant à un domaine, accompagnés de leurs équivalents dans une ou plusieurs autres langues, et qui ne comporte pas de définitions.» 
Par ailleurs, les lacunes des dictionnaires dans les domaines techniques et scientifiques expliquent en grande partie le besoin d'études terminologiques à notre époque de technicité avancée. En effet, les dictionnaires ne comprennent pas toute la gamme de termes du vocabulaire courant, devenus techniques et scientifiques, ni les unités terminologiques dont le sens n'est pas évident pour le non-spécialiste. Prenons un exemple au hasard : nous pouvons constater que le «faisceau laser», dont les caractéristiques permettent diverses applications dans les domaines scientifique et militaire, ne figure pas comme tel dans les entrées des dictionnaires. Certes, le dictionnaire encyclopédique parle de «faisceau» en optique, mais il ne va pas jusqu'à préciser une notion dans un sous-domaine de l'optique. Par contre, nous trouvons l'équivalent anglais ( «laser beam») dans un ouvrage général, le Dictionary of Scientific and Technical Terms, publié chez McGraw-Hill.

Nous remarquons également que les dictionnaires français ne semblent pas contenir autant de termes spécialisés que les dictionnaires anglais. Prenons un autre exemple : «acaricide» se retrouve dans tous les ouvrages généraux anglais, parfois avec une variante «acaracide», parfois avec un synonyme «miticide» (The American Heritage Dictionary of the English Language, the Random House Dictionary of the English Language, Webster's Third New International Dictionary, etc.). Par contre, les ouvrages généraux français n'en font pas mention (ni le Quillet, ni le Grand Larousse encyclopédique, ni le Robert, etc.).

D'une façon générale, il semble que les ouvrages américains comme le Webster's Third New International Dictionary, le Dictionary of Scientific and Technical Terms ${ }^{4}$, présentent plus d'entrées dans les domaines de pointe que les ouvrages généraux français.

En outre, certains ouvrages comme le Vocabulaire systématique de la terminologie 5 et le Petit Robert de 1977 affirment que la terminologie est aussi une «étude systématique des «termes» ou mots et syntagmes spéciaux servant à dénombrer classes d'objets et concepts $[\ldots]^{6}$ " (définition qui pourrait également s'appliquer à la lexicographie). Or, n'oublions pas que l'étude des termes n'est pas obligatoirement systématique en terminologie. («Systématique» est pris ici dans le sens donné à «système» par le Littré (Dictionnaire de la langue française) de 1963: "Doctrine à l'aide de laquelle on dispose et coordonne toutes les notions particulières.») Elle l'est certainement en recherche thématique, mais non pas en recherche ponctuelle, qui est l'étude de problèmes terminologiques isolés. La recherche ponctuelle demeurera nécessaire tant que les progrès techniques continueront à se faire au rythme que nous connaissons depuis plusieurs décennies. Dans un réseau de terminologues spécialisés, les

4. Notons, toutefois, qu'il faut parfois se méfier des synonymes figurant dans ce dictionnaire. En comparant les termes dans des livres spécialisés ou encore dans d'autres ouvrages généraux, nous nous rendons compte que les synonymes cités ne sont pas toujours de vrais synonymes, mais des termes ayant des liens de générique à spécifique ou des notions voisines.

5. Op. cit.

6. Petit Robert, 1977 , p. 1946. 
ponctuelles pourront être transmises aux terminologues, en fonction de leurs domaines de spécialisation.

Remarquons que certains ouvrages traitant de terminologie ont parfois tendance à négliger la recherche ponctuelle. Par exemple, le cahier rédigé avec beaucoup de sérieux par l'Office de la langue française, intitulé Méthodologie de la recherche terminologique ${ }^{7}$, n'accorde que peu de place à la recherche ponctuelle en terminologie. Or la méthodologie en recherche ponctuelle est très importante, notamment en terminologie comparée. Elle consiste, en gros, en diverses «étapes » de consultation : sources écrites d'abord (ouvrages généraux, puis spécialisés), ensuite sources orales au besoin, dans le but de trouver une solution précise à un problème donné.

Autre constatation, les linguistes insistent toujours sur l'importance de la notion en terminologie. Ils partent toujours de la notion pour «nommer». Maillot, par exemple, dans La traduction scientifique et technique de 1970, dit : "La terminologie [...] est une science qui s'attache avant tout à identifier un terme en décrivant la notion qu'il représente $[\ldots]^{8}$. Cela demeure surtout vrai en terminologie unilingue. Si le point de départ est, la plupart du temps, la notion à décrire, il s'agit souvent d'une notion déjà décrite qu'il faut retrouver dans une documentation spécialisée ou nommer dans un autre langue. (C'est le cas, par exemple, d'un appareil inventé aux États-Unis et baptisé par des Américains. La notion est connue, mais il faut trouver dans une langue (ou des langues) la façon de l'appeler.) Nous pouvons aussi partir, non pas de la notion, mais d'un terme pour décrire une notion dans une autre langue. C'est le plus souvent le cas en terminologie comparée. Nous avons donc deux possibilités: soit, partir de la notion pour aboutir au terme (démarche linéaire); soit, partir d'un terme dans une langue donnée pour remonter à la notion, et, de cette notion, aboutir à un terme équivalent dans une autre langue (démarche qui pourrait être représentée par un triangle, comme le dit M. Darbelnet).

DoRothy NAKos-AUPETIT

7. Pierre Auger et Louis-Jean Rousseau, Méthodologie de la recherche terminologique dans Études, Recherches et Documentation, Services des travaux terminologiques de l'Office de la langue française, $\mathrm{n}^{\circ}$ 9, Éditeur officiel du Québec, 1977.

8. Il continue : «sans recourir à des moyens accessoires, tels qu'une illustration, un synonyme, son équivalent dans une autre langue, en somme à en donner la définition. " Or il ne fait maintenant aucun doute que le terminologue doit s'intéresser à tous ces moyens qui pourront l'aider à mieux cerner la notion. 